\title{
Development of the preventive maintenance system for belt conveyors reducers
}

\author{
Boris Gerike ${ }^{1,2,}$, Ivan Panachev ${ }^{2}$, Eugene Kuzin ${ }^{3}$ \\ ${ }^{1}$ Federal Research Center of Coal and Coal Chemistry SB RAS, 650003, 10 Leningradsky av., \\ Kemerovo, Russian Federation \\ ${ }^{2}$ T.F. Gorbachev Kuzbass State Technical University, 650000, 28 Vesennyaya st., Kemerovo, Russian \\ Federation \\ ${ }^{3}$ Branch of T. F. Gorbachev Kuzbass State Technical University in Prokopievsk, 653039, Kemerovo \\ region, 19a Nogradskaya str., Prokopievsk, Russia
}

\begin{abstract}
Heavy operating conditions of mining machines as well as the high level of dynamic loads lead to reduction of their service life. The quantitative estimation of the machine reliability by one of the feature service life - has become widely distributed in all the branches of engineering. Technical diagnosis is one of the important methods of improving the reliability in operating conditions. The diagnostics subsystem should provide for: non-destructive inspection of a technical condition of objects, the definition of sudden and parametric failures of mining machines and their systems, the detection of gradual failures by predicting changes in the monitored parameters, a continuous and periodic technical inspection. The obtained results given in this article prove the possibility of creating a group of common diagnostic criteria suitable for assessing the technical state of reducers of mining machines and equipment, but also being a prerequisite for the effective short-term prediction of the parameters under study when developing adaptive mathematical models.
\end{abstract}

\section{Introduction}

All the parts of mining machines and equipment can be subdivided into the following groups:

1. The first group includes dipper teeth and cutting lips; bases and canopies of powered supports; augers; bulldozer bars, blades; crawler shoes, idler rollers, pins, bushings, sprockets of crawler undercarriage and other parts with the service life duration depending on abrasive wear;

2. The second group includes slotted and threaded parts, gear couplings, mounting seats for rolling bearings of shafts, machine tools, surfaces of gearing, etc. with the service life duration determined by mechanical wear of their surfaces;

\footnotetext{
*Corresponding author: gbl_42@mail.ru
} 
3. The third group includes parts of internal combustion engines of dump trucks, bulldozers, scrapers, diesel locomotives, thermal rock fracturing and jet drilling rigs, etc. with their service life duration limited by molecular - chemical or corrosive mechanical wear;

4. The fourth group includes rolling and plain bearings, shock absorbers, springs, connector rods, connecting rod bolts, etc. with their surface life duration depending on metal fatigue strength.

\section{Formulation of the Problem}

At present, the coal mines operate a significant number of belt conveyors [1, 2], and the performance indicators of the entire coal industry of Kuzbass depend on their operating condition. In the nearest future, it is expected that power consumption and technical equipping of belt conveyors increase, as well as the performance and length of rock mass transportation, variable frequency drives are widely introduced.

The increasing volumes of coal production by integrated mechanized coal faces, together with an increase in production safety require the creation of reliable transport systems. High efficiency and trouble-free performance along with reduction of energy consumption present the main challenge faced by manufacturers of production lines of mine belt conveyors. Another no less important goal is reduction of the cost of their maintenance and repairs $[3,4]$. To ensure reliable operation of a belt conveyor for as long as possible, it is required to find the causes of failure of the various constituent elements [57]. The analysis of downtime caused by failure of a belt conveyor reducer shows that it varies from $7.4 \%$ to $18.2 \%$ and accounts on average for $12 \%$, with the average recovery time is from 24 to 48 hours. It should be noted that the most common failure is belt rupture (up to $50 \%$ ), while the average time to fix this failure is from 1.5 to 2 hours. Hence, identification of the actual technical condition of mine belt conveyor reducers is really quite an urgent objective.

\section{Results of Study}

The vibration control method [8-10] proved to be useful in checking the technical condition of mechanical equipment. The vibration diagnostics is used to monitor the current state of equipment, to identify possible defects, to assess the remaining service and to define repair time and scope. The analysis of domestic and foreign experience of monitoring the technical condition of systems with rotational motion of power assemblies shows that for the detection of potential failures the most effective (77\%) is control of the machinery condition by vibration parameters [11], and with the involvement of other methods of the functional diagnostics - like spectral oil analysis [12] and thermal control, the accuracy of recognition of the cause of the defect increases to $95 \%$.

The functional diagnostics, in terms of safe operation of mining machinery, should play a key role in the field of research and development, production and quality control of the process equipment. And while in the coal mining and ore mining industries the forms of technical service of technological equipment based on its actual condition $[1,8]$ are becoming more common, in the manufacture of such equipment new forms of quality control are not yet implemented in spite of the introduction of quality standards GOST ISO 9000-2011. Defects that arise in the gearbox manufacturing process can be divided into errors in the manufacture of gearbox elements and errors in gearbox assembly.

A full analysis of the technical condition of the gearbox after assembly and trial running on the test bench will allow us to identify and localize manufacturing defects, but also to 
eliminate possible supply of poor quality products to the consumer. In addition, the findings could form the basis for development of automated quality control system.

The analysis of vibration control methods allows us to conclude that it is appropriate to apply the method of support masks for automation of control of products manufactured for the coal industry. This method is based on the fact that the defects formed as a result of assembly operations generate a vibration in certain frequency bands with a certain ratio of the values of controlled parameters. The method of support masks makes it possible to set the width of the frequency band, its position and the values of the evaluation criteria, which are compared with the current values in random order. Based on the analysis of the controlled parameter changes in the frequency band (the number of bands may vary from 6 to 30), the condition of the equipment is evaluated and forecasted [4].

Frequency ranges of the spectrum mask (band width) are usually taken based on the following conditions:

1. "High-energy" components of the spectrum accompanying the misbalance or misalignment $-(0,5 \ldots 1,5) \times f_{r}$ and $(1,5 \ldots 2,5) \times f_{r}$;

2. "Low-energy" components of vibrations accompanying the defects of rolling bearings $-(7,5 \ldots 15,5) \times f_{r}$;

3. $(2,5 \ldots 10,5) \times f_{r}$ - total failure of the system stiffness;

4. The first medium frequency band $(3 \ldots 15) \times f_{r}$;

5. The second medium frequency band $(15 \ldots .40) \times f_{r}$;

6. The first high frequency band $40 \times \mathrm{fr} . . .20 \mathrm{kHz}$;

7. $(0,1 \ldots 0,9) \times f_{r}-$ for detection of oil wedge defects of slide bearings;

8. $(n \pm 1) \times f_{r}$ - for detection of damages in the components of couplings.

The use of modern automation technologies for control of equipment technical condition enables an individual approach to each manufactured mechanism in the evaluation of its technical condition and sets the thresholds of the initial operable and limit state. As an example, Figures 1 and 2 show the spectrum of the vibration signal and its averaged spectral mask in 1 checkpoint of gearbox RKC-400 manufactured by JSC "Anzheromash" (Fig. 3). The measurements were made by analyzers "Corvet", and the signal was processed on Safe Plant software platform developed by NGO "Diatekh".

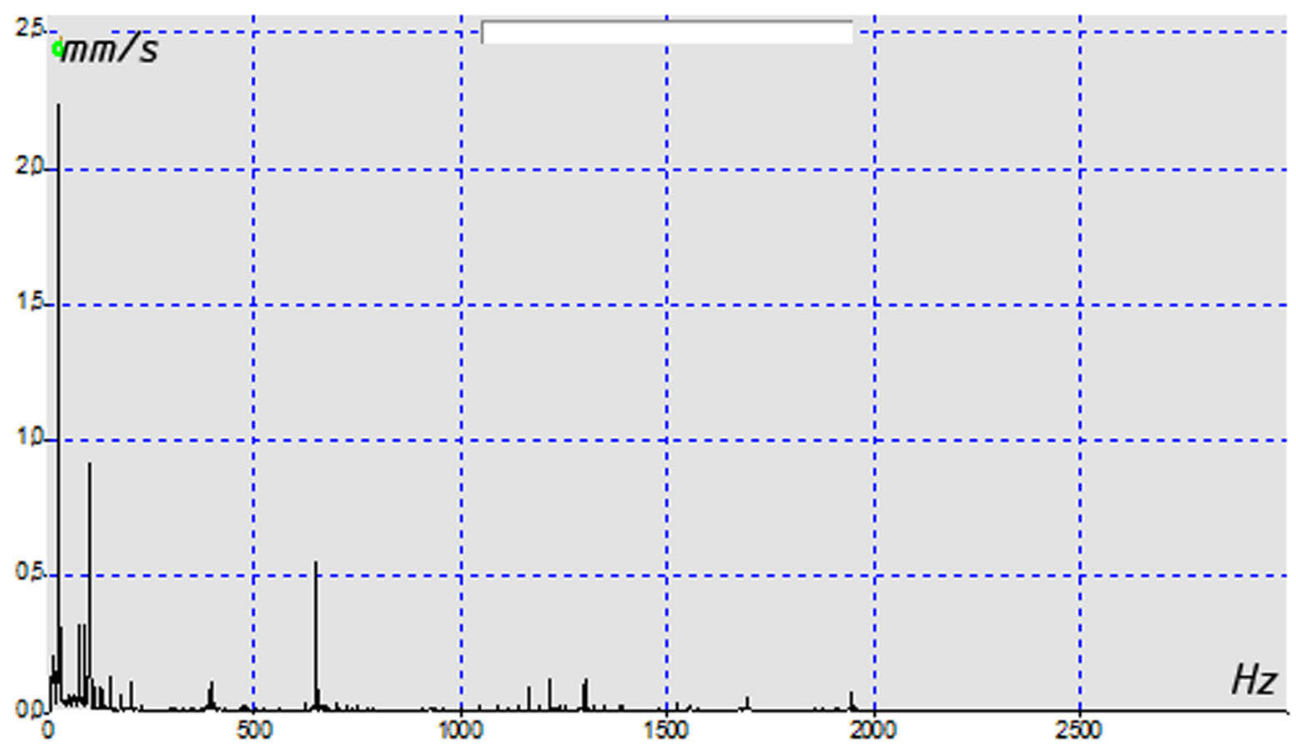

Fig. 1. Vibration signal spectrum 


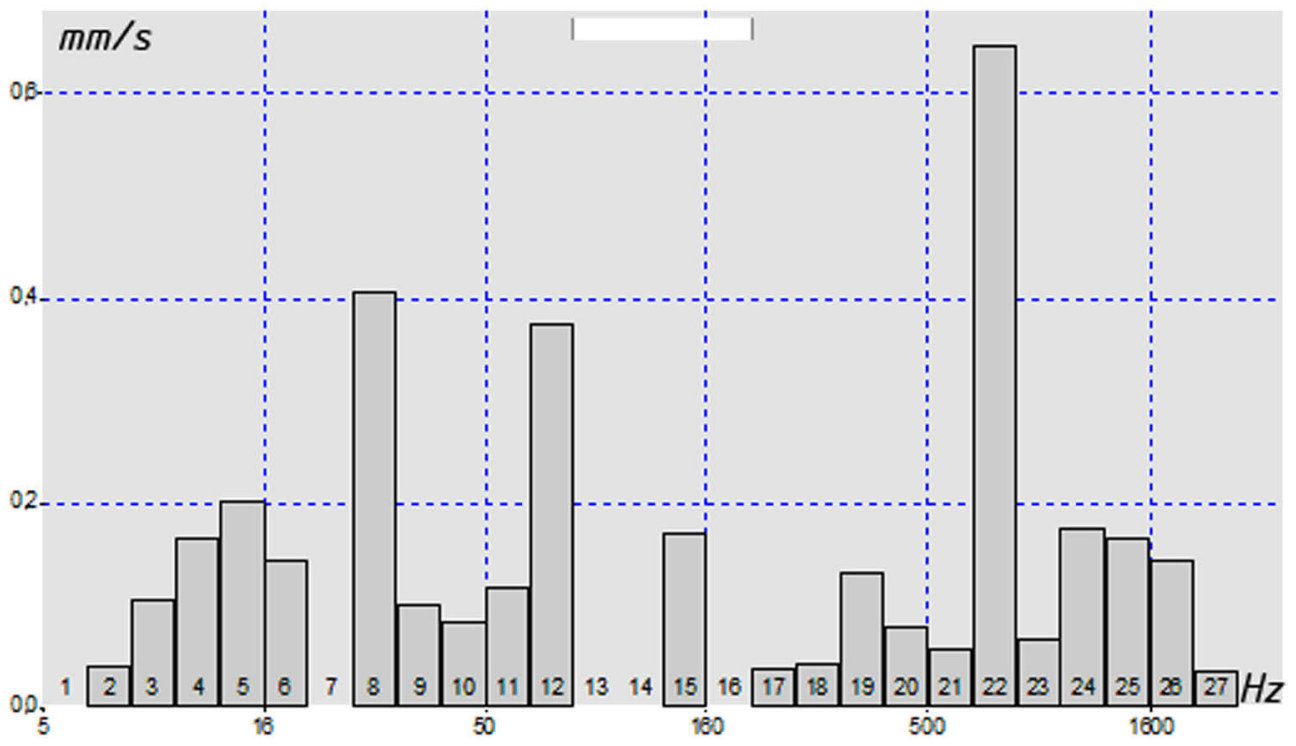

Fig. 2. Vibration signal spectrum mask

In the presented example, the frequency range $(2 ; 3000 \mathrm{~Hz})$ is divided into 27 bands, each of which is normalized by the RMS value of vibration speed $V$ determined for both forward and reverse rotation of the output shaft.

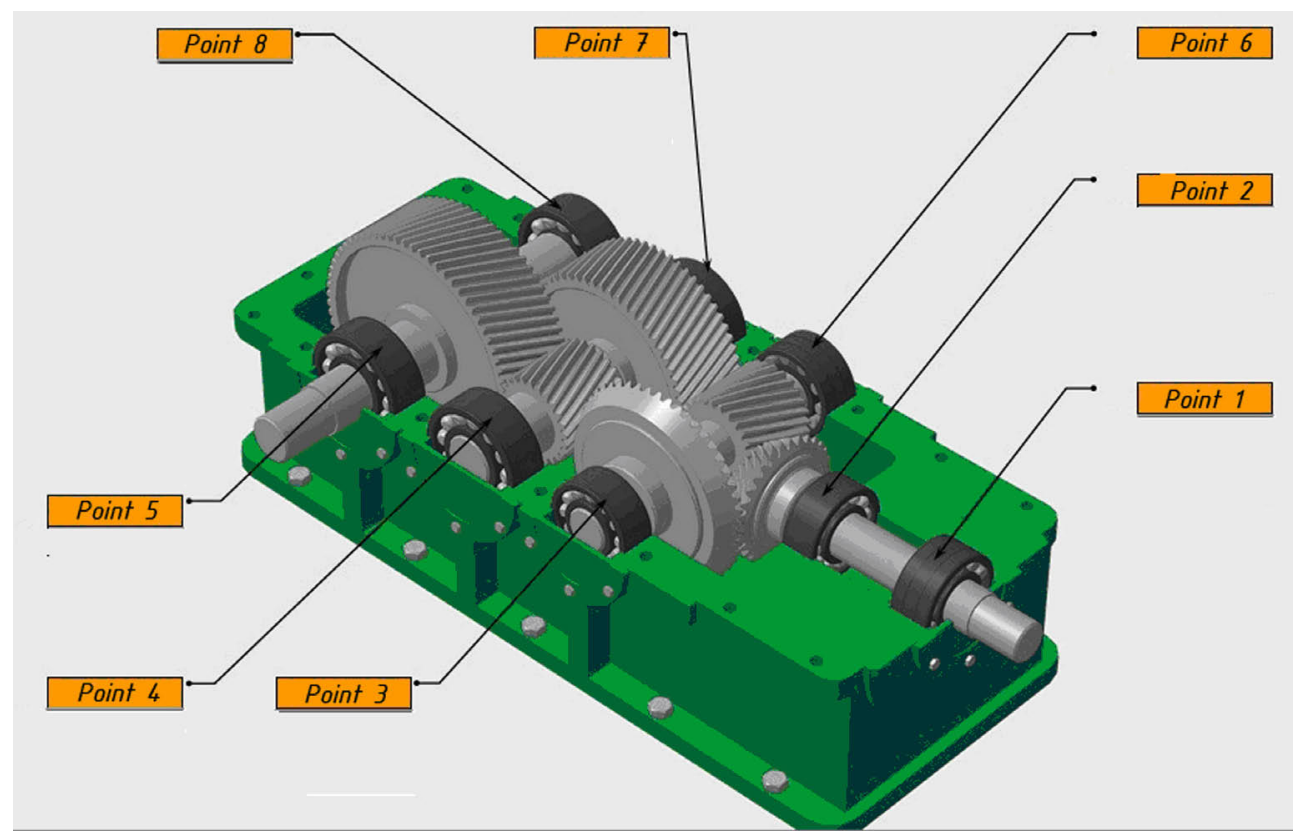

Fig. 3. Vibration measurement points on gearbox RKC-400

The most typical defects inherent to reducers of mining machines occur at frequencies shown in Tables 1 and 2. 
Table 1. Frequencies typical for gearing defects in in-line reducers

\begin{tabular}{|l|l|l|l|}
\hline \multicolumn{1}{|c|}{ Frequency } & \multicolumn{1}{|c|}{$\begin{array}{c}\text { Type of manufacturing } \\
\text { defect }\end{array}$} & $\begin{array}{c}\text { Type of assembly } \\
\text { defect }\end{array}$ & $\begin{array}{l}\text { Type of wear } \\
\text { defect }\end{array}$ \\
\hline$f_{r}$ & Misbalance & & \\
\hline $\begin{array}{l}k \times f_{r 1 \text { and }} k \times f_{r 2} \\
(k=1,2, \text { less often } 3 \text { and } 4), \\
m \times f_{z} \pm n \times f_{r} \\
(m, n=1,2 \ldots)\end{array}$ & Variable gear pitch error & $\begin{array}{l}\text { Violation of } \\
\text { alignment } \\
\text { (misalignment of } \\
\text { shafts) }\end{array}$ & \\
\hline $\begin{array}{l}k \times f_{r} \\
k=1,2 \ldots 20 \text { and higher }\end{array}$ & & $\begin{array}{l}\text { Increased gear } \\
\text { backlash }\end{array}$ & \\
\hline$f_{z}$ & Permanent gear pitch error & & \\
\hline $\begin{array}{l}k \times f_{z}, k \times f_{r} \\
\text { growth of noise component } \\
m \times f_{m} \pm n \times f_{r} \\
(m, n=1,2 \ldots)\end{array}$ & & & Abrasive wear \\
\hline $\begin{array}{l}k \times f_{r}, m \times f_{z} \pm n \times f_{r}, \\
m \times f_{m} \pm n \times f_{r} \\
(\text { amplitude fluctuation, } \\
n=0,1,2 \ldots)\end{array}$ & & & Teeth chipping \\
\hline $\begin{array}{l}k \times f_{r}, \\
m \times f_{z} \pm n \times f_{r}, \\
m \times f_{m} \pm n \times f_{r} \\
(\text { amplitude fluctuation, } \\
n=0,1,2 \ldots), \\
\text { growth of noise component }\end{array}$ & & & \\
\hline
\end{tabular}

Table 2. Defects in the gear part of the planetary reducer and their key diagnostic parameters

\begin{tabular}{|l|l|}
\hline \multicolumn{1}{|c|}{ Type of defect } & \multicolumn{1}{c|}{ Diagnostic signs } \\
\hline Sun pinion shaking & $f_{o}, n f^{*} \pm f_{o}, k f_{z} \pm f^{*}$ \\
\hline Sun pinion misalignment & $2 f_{o}, 2 n f^{*} \pm 2 f_{o}, k f_{z} \pm 2 f^{*}$ \\
\hline Sun pinion teeth defect & $k n f^{*} \pm k_{1} f_{o}, k f_{z} \pm k_{l} f^{*}$ \\
\hline Satellite gear misalignment & $4 f_{g} \pm k_{1} f_{v}, k f_{z} \pm 2 f_{g}$ \\
\hline Satellite gear teeth defect & $2 k f_{g} \pm k_{1} f_{v}, k f_{z} \pm k_{l} f_{g}$ \\
\hline Crown misalignment & $2 n f_{v}, k f_{z} \pm 2 n f_{v}$ \\
\hline Crown teeth defect & $k n f_{v}, k f_{z} \pm k_{1} n f_{v}$ \\
\hline Engagement defect & $k f_{z}$ \\
\hline Shaking of final drive carrier & $k f_{v}, f_{o} \pm f_{v}, k f_{z} \pm k_{1} f_{v}$ \\
\hline Satellite gear bearing defect & $k f_{v}, f_{o} \pm f_{v}, k f_{z} \pm f_{g} / 2$ \\
\hline Defect of sun pinion bearing & $k f_{r}+$ growth of RMS MF, impact pulses appear at MF \\
\hline Bearing lubrication defect & Impact pulses appear at HF, growth of RMS at HF \\
\hline
\end{tabular}

In industrial conditions (mine Taldinskaya-Zapadnaya of OJSC "SUEK-Kuzbass") an integrated method of the technical state estimation has been tested on the drive units of mine belt conveyor 3LL1600 (conveying length $\mathrm{L}=850 \mathrm{~m}$, technical performance $\mathrm{Q}=$ $3500 \mathrm{~m} / \mathrm{h}$, the belt speed $\mathrm{v}=0-4 \mathrm{~m} / \mathrm{s}$ ) by the parameters of the lubricating oil, vibration and thermal control. 


\section{Conclusion}

The proposed approach to the standardization of mechanical vibration parameters can be used in practice at development of the enterprise standard for normalization of vibration of manufactured products for inclusion in the product data sheet.

The development of a large number of spectrum masks for a wide standard line of mining equipment is one of the prerequisites for release by mining machinery plants of quality products and the transition to new forms of mining machines maintenance and repair.

The results of evaluation of the technical condition of the 3LL1600 conveyor drives using the integrated method based on monitoring of parameters of vibro-acoustic signals, emission-spectral analysis of the composition of working oil and thermal visual control of support units of rolling bearings make it possible to track changes in the technical condition of the reducer elements depending on its load and speed.

The proposed approach will not only increase the accuracy of the assessment of the technical condition of mining equipment gear units but will also help to organize the work on creation of normative and methodological base for building predictive models of changes in the technical condition on the basis of a significant amount of accumulated statistical information on the development of those or other defects of belt conveyor reducers.

\section{References}

1. Ansari F., Uhr P. and Fathi M., International Journal of Services, Economics and Management, 6, 14 (2014)

2. Ierace S., Cavalieri S., Management and Production Engineering Review, 4, 37 (2013)

3. Shafiei-Monfared S. and Jenab K., IEEE Transactions on Engineering Management, 59, 293 (2012)

4. Almgren T., Andréasson N., Palmgre M., Patriksson M., Strömberg A.B., Wojciechowsk A. and Önnheim M., Proceedings of 4th World Conference on Production \& Operations Management and the 19th International Annual EurOMA Conference, University of Amsterdam (Amsterdam, 2012)

5. Franco Jefferds dos Santos Silva, Herbert Ricardo Garcia Viana, André Nasser Aquino Queiroz, Journal of Quality in Maintenance Engineering, 22, 418 (2016)

6. Gajanand Gupta, Rajesh P Mishra, Journal of Quality in Maintenance Engineering, 22, 130 (2016)

7. Dehghanian P. and Aminifar F., IEEE Transactions on Power Delivery, 28, 761 (2013)

8. Prabhakar D. and Raj V.P., International Journal of Scientific \& Technology Research, 2, $56(2013)$

9. Anil Rana, Journal of Quality in Maintenance Engineering, 22, 180 (2016)

10. Golmakani H.R. and Morteza P., J of Quality in Maintenance Eng., 20, 51 (2014)

11. Hussan Saed Al-Chalabi, Jan Lundberg, Majid Al-Gburi, Alireza Ahmadi, Behzad Ghodrati, Journal of Quality in Maintenance Engineering, 21, 207 (2015)

12. Hartman J.C. and Tan C.H., The Engineering Economist, 59, 136 (2014)

13. Hussan S. Al-Chalabi, Jan Lundberg, Andi Wijaya, Behzad Ghodrati, Journal of Quality in Maintenance Engineering, 20, 306 (2014)

14. Gustafson A., Schunnesson H., Galar, D. and Kumar, U., International Journal of Mining, Reclamation and Environment, 27, 75 (2013) 